\title{
Association between LDL-C/HDL-C Ratio and Carotid Atherosclerosis in an Asymptomatic Japanese Population: A Cross-Sectional Study
}

\section{Juan Wang}

The Fifth Affiliated Hospital of Zhengzhou University

Jiuling Liu ( $\sim$ ljl352690@163.com )

Nanjing BenQ Medical Center https://orcid.org/0000-0002-7896-5211

\section{Research}

Keywords: Carotid atherosclerosis, Carotid plaque score, LDL-C/HDL-C ratio

Posted Date: October 26th, 2021

DOI: https://doi.org/10.21203/rs.3.rs-994711/v1

License: (c) (i) This work is licensed under a Creative Commons Attribution 4.0 International License. Read Full License 


\section{Abstract}

Background and aims LDL-C/HDL-C ratio predicted atherosclerosis progression better than LDL-C or HDL-C alone. However, the association between LDL-C/HDL-C ratio and Carotid Atherosclerosis(CA) is still controversial. There is a lack of research on this topic in theAsymptomatic Japanese Population. This study aims to provide further results. Methods The study population was a cross-sectional study of 1904 subjects free of cardio-cerebrovascular disease at baseline(mean age $57 \pm 11.9$ years, $51.9 \%$ male). All participant sultrasonography of the carotid artery. The presence of carotid plaque score(PS) aand plaque number (PN) were evaluated by ultrasonography. Multivariate logistic regression models to estimatethe LDLC/HDL-C ratio and PS relationship. Participants were stratified into three groups based on LDL-C/HDL-C ratio tertiles. Interaction and stratified analyses were conductedaccording to age, sex, smoking status, drinking status, fatty, and histories of diabetes. Results In regression models and after multiple adjustments, the risk of PS was significantly associated with serum LDL-C/HDL-C ratio levels in which LDL-C/HDL-C ratio was included as a categorical variable. It remained significant for the highest vs the first tertile of the LDL-C/HDL-C ratio $(O R=1.50,95 \% \mathrm{Cl} 1.04-2.17)$. Stratified analysis, we found that the association was more significant aged $<65$ years old, female and non-diabetes subgroups. Interaction analysis showed no interaction between LDL-C/HDL-C ratio and PS in the fatty,smoking, and drinking subgroups. Conclusions In conclusion, LDL$\mathrm{C} / \mathrm{HDL}-\mathrm{C}$ ratio is an independent risk factor for $\mathrm{CA}$ in the Japanese population. A prospective and randomized clinical trial of LDL-C/HDL-C ratio lowering therapy in the Japanese population is needed to assess the causal nature of the relationship.

\section{Introduction}

Atherosclerosis is well known to contribute to the occurrence and development of ischaemic cardiovascular disease (CVD) and acute ischemic stroke(AIS)[1-3]. In 2020, the prevalence, prevalence and risk factors of carotid atherosclerosis in populations in 21 countries and regions were assessed,atherosclerosis is a global public health problem affecting nearly two billion people[4]. The early detection of atherosclerosis in asymptomatic population mainly focuses on peripheral arteriesand carotid arteries; The carotid artery is shallow because of its position and a 'window' for systemic arterial disease[5]. Studies suggest that Carotid Plaques are reliable markers of carotid atherosclerosis[6]. Zhao et al.[7] found that aopproximately $30 \%$ of first acute cardiovascular events without any clinical symptoms are fatal. Therefore, it is necessary to identify the risk factors for the silent preclinical stage of atherosclerosis to prevent CVD and AIS.

Previous studies have shown that LDL-C and HDL-C have been widely used to monitor lipid-lowering indexes in almost all CVD patients[8]. However, some clinical trials have shown that despite this, $60-70 \%$ of CVD events continue to occur[9-11]. Increasing evidence points to the LDL-C/HDL-C ratio as a novel indicator of the risks of both atherosclerotic cardiovascular and cerebrovascular diseases, as it simultaneously takes into account both LDL-C and HDL-C ratio levels[12-14]. Some studies have suggested that the LDL-C/HDL-C ratiois positively associated with CVD[14-16], while others have found a negative correlation between the LDL-C/HDL-C ratio and all-cause mortality[17-19]. However, previousstudies on the LDL$\mathrm{C} / \mathrm{HDL}-\mathrm{C}$ ratio have drawn inconsistent conclusions.

In the present study, PS is assessed as indicators of CA to investigate the association between LDL-C/HDL-C ratio and CA in an Asymptomatic Japanese Population. It also was attempted to indicate how vascular risk factors may influence the mentioned association with the assistance of stratified analysis.

\section{Materials And Methods}

\subsection{Design and participants}

The original clinical data were derived from a public dataset (https://datadryad.org) offered by Drs, which was described in detail previously[20]. It conformed to the guidelines of theDeclaration of Helsinki and was approved by the approval of the ethical review committee of Shin Takeo Hospital. We also made an effort to cite the Dryad data package following protocols released in the Dryad Digital Repository (Tokyo, Japan). Briefly, the study was a Cross-Sectional study of the individual who 
participated in the medical examination programat Shin Takeo Hospital from April 1st, 2016 to Oct 31st, 2017[20]. Some patients underwent blood tests and carotid ultrasonography,and completed standard questionnaires all within this period. Eventually, 1904 asymptomatic participants, including 988 males and 916 females were included in this study ${ }^{[20]}$.

\subsection{Data Collection And Measuremen}

Data collection and measurement has been described previously[20]. The general inspection (age, sex, body mass index, diastolic blood pressure[DBP], systolic blood pressure [SBP]), medical history, longterm medication use (antihypertensive medicine, antidiabetic medicine, and cholesterol-lowering medications), and lifestyle(including smoking and drinking habits), of partic-ipants were obtained by using a standardized self-administered questionnaire. Blood samples were drawn from the antecubital vein of seated participants after $8 \mathrm{~h}$ of fasting. Fasting blood samples were analyzed for LDL-C, HDL-C, LDL$\mathrm{C} / \mathrm{HDL}-\mathrm{C}$ ratio, triglyceride, fasting plasma glucose, and HBA1c. The smoking status was categorized intotwo groups: Nonsmokers and smokers. Non-smokers were referred to the participants who never smoked cigarettes. Smokers were defined as individuals who had smoked in the past but quitted smoking until baseline or as participants who smoked at baseline. Drinking status (rarely, sometimes, and daily), and drinking volume (with respect to quantify the consumption of alcoholic beverage/day) in form of $<180,180-360,360-540$ or $>540 \mathrm{~mL}$. Hypertension was defifined as a history of hypertension or $\mathrm{SBP} \geq 140$ or DBP $\geq 90 \mathrm{mmHg}$ (or both) or oral hypotensive agents. Diabetes was defined either by fasting plasma glucose $(F P G) \geq 7.00 \mathrm{mmol} / \mathrm{L}$, and/or self-reported history of diabetes, and/or treatment with insulin or oral hypoglycemic agents duringthe follow-up period.

\subsection{Carotid Artery Color Doppler Ultrasound}

The ultrasonic testing was conducted with ultrasonic diagnostic equipments, LOGIQ S7 Expert (GE Healthcare Japan, Tokyo) and Aplio 400 (Canon Medical systems, Tochigi, Japan). Two characteristics were recorded: carotid plaque score (PS)[21] and plaque number (n-plaque). The PS was calculated as follows. The carotid artery was divided into four $15 \mathrm{~mm}$ long sections: the central side of the common CA, the peripheral side of the CA, the bifurcation of the CA and the central side of the internal carotid artery. Then, the sum of the maximum values of intima-media thickness exceeding $1.0 \mathrm{~mm}$ was calculated[22].To measure both the left and right carotid arteries by experienced radiologists blinded to the clinical data.

\section{Statistical analysis}

Baseline characteristics of the study population were divided into three groups based on LDL-C/HDL-C ratio tertiles(T1-3) : $0.3 \leq \mathrm{T} 1<1.6,1.7 \leq \mathrm{T} 2<2.3,2.4 \leq \mathrm{T} 3<5.5$. Continuous variables are presented as the mean (standard deviation (SD)) or median (range) if non-normally distributed, categorical variables were presented as frequency (percentage). The association of LDL$\mathrm{C} / \mathrm{HDL}-\mathrm{C}$ ratio and PS was assessed with the use of multivariable logistic regression models. In another separate analysis, LDL-C/HDL-C ratio was included as a continuous variable in the model. Four different models were constructed in this study and results were presented as both crude and adjusted hazard ratios (OR) estimates with $95 \% \mathrm{Cl}$. The potential confounders included in this study was selected based on their associations with the outcome or a change in effect estimate of more than $10 \%$. Model 1 was adjusted for age, sex, history of diabetes, and history of hypertension at baseline. Model 2 was adjusted for covariates included in Model 1, plus BMI, somking habits, medication to reduce a level of cholesterol at baseline. Model 3 was adjusted for covariates included in Model 2 and HBA1C, TG, BS. Interaction and stratifified analyses were conducted according to age $(\varangle 45, \geq 45$ and $<65, \geq 65)$, sex, fatty, diabetes mellitus, smoking habits, driking habits. All subgroup analyses adjusted TG, blood glucose level, systolic blood pressure, diastole blood pressure, medication to reduce a level of cholesterol except the stratification factor itself. All the analyses were performed with the statistical software packages R.(http://www.Rproject.org, The R Foundation) and Free Statistics software versions 1.3. A two-tailed test was performed and $p<0.05$ was considered statistically signifificant. 


\section{Results}

\subsection{Participants' characteristics}

The current study enrolled 988 men and 916 women. Baseline characteristics of participantsby the tertile of the LDL-C/HDL-C ratio are shown in Table 1. In the general inspection,significant differences in sex, BMI, as well as drinking habits, and smoking habits, and the presence of metabolic syndrome, medications to reduce a level of cholesterol were found between

groups. In the blood tests, significant differences were seen in HDL, LDL, HbA1c, BS, SBP and DBP. In the ultrasonic tests, significant differences were found in PS. 
Table1 Baseline characteristics of the study populations by the baseline LDL-C/HDL-C ratio

\begin{tabular}{|c|c|c|c|c|c|}
\hline \multirow{3}{*}{$\begin{array}{l}\text { Baseline } \\
\text { characteristics }\end{array}$} & \multirow{2}{*}{$\begin{array}{l}\text { Total } \\
\mathrm{n}=1904\end{array}$} & \multicolumn{4}{|c|}{ LDL-C/HDL-C ratio } \\
\hline & & Tertile 1 & Tertile 2 & Tertile 3 & $p$ \\
\hline & & $(n=579)$ & $(n=687)$ & $(n=638)$ & \\
\hline
\end{tabular}

\section{General}

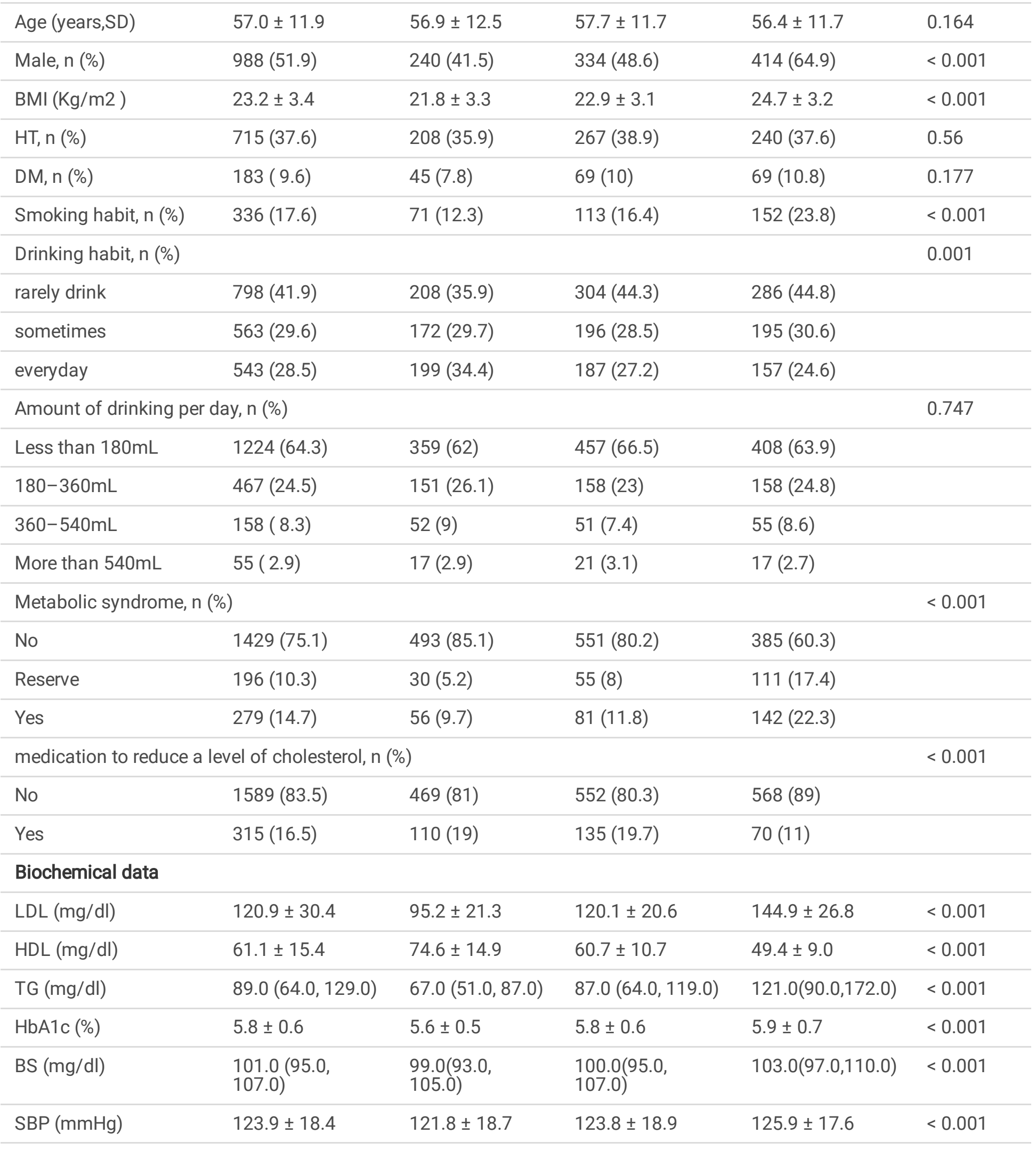




\section{Carotid data}

\begin{tabular}{lllll}
\multicolumn{4}{l}{ The number of plaque, $\mathrm{n}(\%)$} & \\
\hline 0 & $1241(65.2)$ & $405(69.9)$ & $437(63.6)$ & $399(62.5)$ \\
\hline 1 & $337(17.7)$ & $83(14.3)$ & $129(18.8)$ & $125(19.6)$ \\
\hline 2 & $203(10.7)$ & $60(10.4)$ & $72(10.5)$ & $71(11.1)$ \\
\hline 3 & $79(4.1)$ & $19(3.3)$ & $31(4.5)$ & $29(4.5)$ \\
\hline 4 & $33(1.7)$ & $9(1.6)$ & $13(1.9)$ & $11(1.7)$ \\
\hline 5 & $6(0.3)$ & $3(0.5)$ & $1(0.1)$ & $2(0.3)$ \\
\hline 6 & $4(0.2)$ & $0(0)$ & $3(0.4)$ & $1(0.2)$ \\
\hline 8 & $1(0.1)$ & $0(0)$ & $1(0.1)$ & $0(0)$ \\
\hline PS, n (\%) & $663(34.8)$ & $174(30.1)$ & $250(36.4)$ & $239(37.5)$
\end{tabular}

Values are given as mean \pm standard deviation, medians with interquartile range or number (\%). BMI, body mass index. HT, hypertension. DM, diabetes mellitus. LDL-C, low-density lipoprotein cholesterol. HDL-C, high-density lipoprotein cholesterol. LDL-C/HDL-C ratio, quotient of LDL-C and HDL-C. TG, Triglyceride. HbA1c, hemoglobin A1c. BS, blood glucose level. SBP, systolic blood pressure. DBP, diastole blood pressure. PS, carotid plaque score. PN, plaque number.

\subsection{The relationship between LDL-C/HDL-C ratio and PS in multiple regression model in Table 2}

After adjustment in multivariable analyses, LDL-C/HDL-C ratio was significantly associated with PS. When included as a continuous variable, LDL-C/HDL-C ratio was also associated with an increased risk of PS (adjusted OR per 1-unit increase in LDL-C/HDL-C ratio, $1.20(95 \% \mathrm{Cl}, 1.06-1.36)$. It could be interpreted that a unit increase of LDL-C/HDL-C ratio exhibited a correlation with a $20 \%$ increase of incidence of PS. In the unadjusted model, compared with people in the bottom tertiles, the OR for PS risk was $1.33(95 \% \mathrm{Cl} 1.05-1.69)$ and $1.39(95 \% \mathrm{Cl} 1.1-1.77)$ for individuals with LDL-C/HDL-C ratio in the second, third, respectively. In the model 3, compared with people in the first tertiles, the OR for PS risk was 1.35 (95\% Cl: 1 1.82) and 1.5 (95\% Cl: 1.04 2.17) for individuals with LDL-C/HDL-C ratio in the second, third, respectively. The restricted cubic splines (Fig. 1) showed a signifificant linear association between LDL-C/HDL-C ratio and risk of high PS ( $p$ for non-linearity $=0.805$ ). 
Table2 Association between baseline LDL-C/HDL-C ratio and PS hyperintensity in multiple regression model.

\begin{tabular}{|c|c|c|c|c|c|c|c|c|}
\hline \multirow[t]{2}{*}{ Variable } & \multicolumn{2}{|c|}{ Non-adjusted Model } & \multicolumn{2}{|l|}{ Model-1 } & \multicolumn{2}{|l|}{ Model-2 } & \multicolumn{2}{|l|}{ Model-3 } \\
\hline & $\mathrm{OR}(95 \% \mathrm{Cl})$ & $\begin{array}{l}\mathrm{P} \text { - } \\
\text { value }\end{array}$ & $\mathrm{OR}(95 \% \mathrm{Cl})$ & $\begin{array}{l}\mathrm{P}- \\
\text { value }\end{array}$ & $\mathrm{OR}(95 \% \mathrm{Cl})$ & $\begin{array}{l}\mathrm{P}- \\
\text { value }\end{array}$ & $\mathrm{OR}(95 \% \mathrm{Cl})$ & $\begin{array}{l}\mathrm{P} \text { - } \\
\text { value }\end{array}$ \\
\hline $\begin{array}{l}\text { LDL- } \\
\text { C/HDL-C } \\
\text { ratio per } 1 \\
\text { mg/dL }\end{array}$ & $\begin{array}{l}1.2 \\
(1.06 \sim 1.36)\end{array}$ & 0.004 & $\begin{array}{l}1.23 \\
(1.07 \sim 1.42)\end{array}$ & 0.004 & $\begin{array}{l}1.31 \\
(1.12 \sim 1.52)\end{array}$ & 0.001 & $\begin{array}{l}1.37 \\
(1.12 \sim 1.68)\end{array}$ & 0.002 \\
\hline \multicolumn{9}{|l|}{$\begin{array}{l}\text { LDL- } \\
\text { C/HDL-C } \\
\text { ratio } \\
\text { Tertiles }\end{array}$} \\
\hline $\begin{array}{l}\text { LDL- } \\
\text { C/HDL-C } \\
\text { ratio1( } \geq 0.3, \\
\otimes 1.6)\end{array}$ & 1 & & 1 & & 1 & & 1 & \\
\hline $\begin{array}{l}\text { LDL- } \\
\text { C/HDL-C } \\
\text { ratio2( } \geq 1.7, \\
₫ 2.3)\end{array}$ & $\begin{array}{l}1.33 \\
(1.05 \sim 1.69)\end{array}$ & 0.017 & $\begin{array}{l}1.29 \\
(0.99 \sim 1.69)\end{array}$ & 0.059 & $\begin{array}{l}1.32 \\
(1 \sim 1.72)\end{array}$ & 0.046 & $\begin{array}{l}1.35 \\
(1 \sim 1.82)\end{array}$ & 0.053 \\
\hline $\begin{array}{l}\text { LDL- } \\
\text { C/HDL-C } \\
\text { ratio3( } \geq 2.4, \\
\bigotimes 5.5)\end{array}$ & $\begin{array}{l}1.39 \\
(1.1 \sim 1.77)\end{array}$ & 0.006 & $\begin{array}{l}1.34 \\
(1.02 \sim 1.76)\end{array}$ & 0.034 & $\begin{array}{l}1.47 \\
(1.1 \sim 1.96)\end{array}$ & 0.009 & $\begin{array}{l}1.5 \\
(1.04 \sim 2.17)\end{array}$ & 0.031 \\
\hline$P$ for trend & & 0.008 & & 0.038 & & 0.01 & & 0.035 \\
\hline
\end{tabular}

Non-adjusted Model.

Model 1: adjusted for age, sex, hypertension, diabetes mellitus at baseline.

Model 2: adjusted for Model $1+$ BMI, smoking habits, medication to reduce a level of cholesterol.

Model 3: adjusted for Model 2 + blood glucose level, triglyceride, HbA1c.

In order to assess the robustness of our results, following subgroup stratified analyses were performed. We further conducted subgroup analyses stratifified by age, sex, fatty, diabetes, smoking habits, drinking habits to estimate the association between LDL-C/HDL-C ratio and risk of PS are presented in Table 3 and Fig 2. The interaction analysis revealed that age,sex, Diabetes could make a link between LDL-C/HDL-C ratio and incidence of PS. Firstly, there was a significant interaction between LDL-C/HDL-C ratio and age $(P=0.002)$, No significant correlation was found between LDL-C/HDL-C ratio and PS in subjects aged $\geq 65$ years old(adjusted $\mathrm{OR}, 0.97 ; 95 \% \mathrm{Cl}, 0.77-1.21$ ). However, the results for female and diabetes subgroups were showing a positive relationship between elevated LDL-C/HDL-C ratio and higher risk of without diabetes, and this association appeared to be more substantial in participants with female. Interaction analysis showed no interaction between LDL-C/HDL-C ratio and PS in the fatty, smoking and drinking subgroups. The stratified analysis demonstrated a statistically significant association between LDL-C/HDL-C ratio and PS in without fatty subjects (adjusted OR, 1.32; 95\% $\mathrm{Cl}, 1.08-1.6$ ), subjects without smoking habits (adjusted OR, 1.23; $95 \% \mathrm{Cl}, 1.04-1.45$ ). It was illuminated that a high LDL-C/HDL-C ratio was positively associated with PS incidence in subjects rarely and sometimes drink, rather than in everyday drink subjects.

Table3 Association between baseline LDL-C/HDL-C ratio and the risk of Carotid Atherosclerosis in the subgroup analyses 


\begin{tabular}{|c|c|c|c|c|c|c|}
\hline Subgroup & Total & PS & $\mathrm{OR}(95 \% \mathrm{Cl})$ & & $P$ value & $P$ value for interaction \\
\hline & $n(\%)$ & $n(\%)$ & No adjusted & adjusted & & \\
\hline Age & & & & & & 0.002 \\
\hline$\square 45$ & 343 & $23(6.7)$ & $2.2(1.41 \sim 3.43)$ & $1.97(1.21 \sim 3.2)$ & 0.006 & \\
\hline$\geq 45,065$ & 707 & $200(28.3)$ & $1.64(1.33 \sim 2.02)$ & $1.53(1.21 \sim 1.93)$ & $<0.001$ & \\
\hline$\geq 65$ & 854 & $440(51.5)$ & $0.98(0.81 \sim 1.19)$ & $0.97(0.77 \sim 1.21)$ & 0.775 & \\
\hline Sex & & & & & & 0.003 \\
\hline male & 988 & $413(41.8)$ & $0.92(0.78 \sim 1.07)$ & $0.99(0.83 \sim 1.18)$ & 0.88 & \\
\hline female & 916 & $250(27.3)$ & $1.6(1.29 \sim 1.99)$ & 1.33 (1.03 1.73) & 0.031 & \\
\hline Fatty & & & & & & 0.101 \\
\hline No & 1190 & 392 (32.9) & 1.39 (1.17 1.65) & $1.32(1.08 \sim 1.6)$ & 0.007 & \\
\hline Yes & 714 & $271(38)$ & $0.94(0.78 \sim 1.15)$ & $1.1(0.88 \sim 1.37)$ & 0.409 & \\
\hline Diabetes & & & & & & 0.01 \\
\hline No & 1721 & $554(32.2)$ & 1.25 (1.09 1.42) & $1.24(1.07 \sim 1.44)$ & 0.004 & \\
\hline Yes & 183 & 109 (59.6) & $0.77(0.52 \sim 1.14)$ & $0.73(0.45 \sim 1.18)$ & 0.192 & \\
\hline Smoking habits & & & & & & 0.119 \\
\hline No & 1568 & $541(34.5)$ & $1.27(1.1 \sim 1.47)$ & 1.23 (1.04 1.45) & 0.018 & \\
\hline Yes & 336 & $122(36.3)$ & $1.01(0.78 \sim 1.29)$ & 1.03 (0.77 1.39) & 0.826 & \\
\hline Drinking habits & & & & & & 0.064 \\
\hline rarely drink & 798 & 259 (32.5) & $1.32(1.08 \sim 1.6)$ & 1.35 (1.06 1.72) & 0.013 & \\
\hline sometimes & 563 & 177 (31.4) & $1.39(1.1 \sim 1.76)$ & $1.32(1 \sim 1.73)$ & 0.048 & \\
\hline everyday & 543 & $227(41.8)$ & $1.01(0.81 \sim 1.26)$ & $1.13(0.88 \sim 1.46)$ & 0.351 & \\
\hline
\end{tabular}

Each stratification adjusted for all the factors (Triglyceride, HbA1c, blood glucose level, systolic blood pressure, diastole blood pressure, medication to reduce a level of cholesterol) except the stratification factor itself. 


\section{Discussion}

To the best of our knowledge, this is the first study to describe a cross-sectional relationship between LDL-C/HDL-C ratio and risk of incident carotid atherosclerosis in a population with asymptomatic in Japan. In the present study, the LDL-C/HDL-C ratio was identified as a significant and independent risk factor of PS in the asymptomatic population. Indeed, a unit increase in LDL-C/HDL-C ratio level was associated with a $20 \%$ higher risk of ps, further confirming the relationship between LDL$\mathrm{C} / \mathrm{HDL}-\mathrm{C}$ ratio and PS. Moreover, we found that both age and sex and DM significantly influenced this relationship, suggesting that these three factors may interact with the ps biological pathway to stimulate the development of LDL-C/HDLC ratio.

LDL-C is considered a primary target of lipid-lowering therapy in almost all patients with CVD. However, even LDL-lowering therapy using statin agents still leaves considerable residual risk of CVD events, which may be due to residual risks associated with lipid abnormalities[23, 24]. A prospective community-based cohort study demonstrated that LDL-C/HDL-C was a better predictor of CIMT progression than either HDL-C or LDL-C levels alone 2020[25, 26]. Enomoto M et al.[27]found LDL-C:HDL-C ratio was a better marker in predicting IMT progression than HDL-C or LDL-C alone during an 8-year follow-up survey.

Previous studies indicated that LDL-C:HDL-C ratio is recognised as a new biometric that has clinical relevance in AS

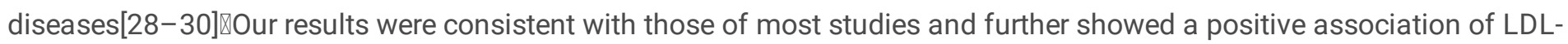
C/HDL-C with presence of carotid plaque in Japanese. In the Helsinki Heart Study analyses, LDL-C:HDL-C ratio was shown to be the best single predictor of cardiac events[31]. Katakami et al.[32]explored the relationships between various lipid parameters including lipid ratios and carotid AS in 934 patients with T2DM having no obvious atherosclerotic diseases in Japan. They concluded that LDL-C:HDL-C ratio was positively associated with carotid atherosclerotic plaques. Wu et al[33] surveyed the impact of LDL-C:HDL-C ratio on CIMT among 1579 residents aged 40-74 years in northern Taiwan. The results demonstrated that LDL-C:HDL-C ratio was an important determinant of increased CIMT. A series of studies have found that LDL-C/HDL-C may provide greater predictive power for atherosclerotic change by concurrently reflecting both cholesterol entering and leaving the arterial intima. However, in some studies, association was not observed between LDL-C/HDL-C with carotid plaque failed to identify any associations between LDL-C:HDL-C ratio and early carotid AS CIMT carotid plaques as well as carotid plaque type[34]. The small sample of thestudy may possibly explain the discrepancy.

Although the association between LDL-C/HDL-C ratio and PS in the stratified analysis was consistent with that in the multivariable logistic regression analysis. We found that it was not statistically significant in subjects older than 65 years, smokers, drinkers and in subjects who were diagnosed with fatty, diabetes. While aging, smoking, drinking, diabetes and fatty are risk factors for ps subjects with these risk factors are also supposed to have higher LDL-C/HDL-C ratio levels in the group. Consequently, residual confoundings were inevitable when we analyzed association of LDL-C/HDL-C ratio and prevalence of PS in subjects with these factors.Additionally, the sample size decreased after stratification,there was no significant association between LDL-C/HDL-C ratio and high CIMT in the subgroup of T2DM, which was similar to the significant association between LDL-C/HDL-C ratio and CIMT in a Cohort-sectional study of 13612 Chiese T2DM patients[35]. T2DM is characterized by a clustering of CVD risk factors including dyslipidemia. Different study design and method of statistical analysis, and a smaller sample size of T2DM $(n=183)$ in our study may explain the discrepancy.

This study found a linear association between LDL-C/HDL-C ratio and the risk of ps by the restricted cubic spine. The significant Ptrendin the Cox model also indirectly indicated the linear association. Our previous work described the cut-off value of LDL-C:HDL-C ratio for assessing CVD risk factors was 2.5 among Uygur adults[7]. However, we observeda less risk between 1.6 and 2.3 but a significant increase for values uper than 2.3, which indicated that keeping the values of LDL$\mathrm{C} / \mathrm{HDL}-\mathrm{C}$ ratio between 1.6 and 2.3 may be the suggested range of the LDL-C/HDL-C ratio to prevent high ps. The discordance between our results may be due to different characteristics between our population and theirs. Moreover, people with the LDL-C/HDL-C ratio $>2.3$ had an increased risk of high ps and the risk of high ps increased gradually with the increase 
in the LDL-C/HDL-C ratio. The finding suggests that maintaining the LDL-C/HDL-C ratio under a low level may be useful to prevent and control the progression to high PS.

\section{Limitations}

However, limitations should also be considered in deriving conclusions. First, although we adjusted for numerous confounding factors, there may be potential residual con-founding factors as with all Cross-Sectional studies. Second, this study included only Japanese participants, and therefore these fifindings may not be generalisable to other biogeo graphic ethnic groups.TIRD, we were unable to observe the long-term changes in the serum lipid levels.

\section{Conclusions}

In summary, our results indicate that the LDL-C/HDL-C ratios have a linear association with carotid atherosclerosis in Japanese. These data suggest that LDL-C/HDL-C ratios are recommended as a tool to assess the risk of earlystage atherosclerosis in regular clinicalpractice.

\section{Declarations}

\section{Ethics statement}

The studies involving human participants were reviewed and approved by the ethical review committee of Shin Takeo Hospital. The patients/participants provided their written informed consent to participate in this study.

\section{Authors' contributions}

Juan Wang and Jiuling Liu contributed equally to this work. Juan Wang participated in the study design, statistical analysis, writing and revising the manuscript. Jiuling Liu participated in the study design and interpretation of data and revising the manuscript. Juan Wang participated in the study design and interpretation of data. Jiuling Liu contributed to statistical analysis and revised the manuscript critically. All authors read and approved the final manuscript.

\section{Funding}

There was no funding for this study.

\section{Availability of data and materials}

The data are available at http://www.Datadryad.org/. which allows researchers to freely download the original data.

\section{Conflict of interest}

The authors declared they do not have anything to disclose regarding conflict of interest with respect to this manuscript.

\section{Acknowledgments}

The authors are very grateful to Jie-Liu, PhD (Department of Vascular and Endovascular Surgery, Chinese PLA General Hospital), for his helpful review and comments regarding the manuscript. Dr. Qilin Yang (The Second Affiliated Hospital of Guangzhou Medical University, Guangzhou, Guangdong, China) for helping in this revision. And Kazuo Ishii, Ph.D (Department of Applied Information Engineering, Faculty of Engineering, Suwa University of Science, JAPAN, thanks to him for providing the data for this article.

\section{References}


[1]. Kalsch, H., et al., Association of progressive thoracic aortic calcification with future cardiovascular events and all-cause mortality: ability to improve risk prediction? Results of the Heinz Nixdorf Recall (HNR) study. Eur Heart J Cardiovasc Imaging, 2019. 20(6): p. 709-717.

[2]. Lehmann, N., et al., Value of Progression of Coronary Artery Calcification for Risk Prediction of Coronary and Cardiovascular Events: Result of the HNR Study (Heinz Nixdorf Recall). Circulation, 2018. 137(7): p. 665-679.

[3]. Zhou, D., et al., Irregular surface of carotid atherosclerotic plaque is associated with ischemic stroke: a magnetic resonance imaging study. J Geriatr Cardiol, 2019. 16(12): p. 872-879.

[4]. Song, P., et al., Global and regional prevalence, burden, and risk factors for carotid atherosclerosis: a systematic review, meta-analysis, and modelling study. Lancet Glob Health, 2020. 8(5): p. e721-e729.

[5]. Piepoli, M.F., et al., 2016 European Guidelines on cardiovascular disease prevention in clinical practice: The Sixth Joint Task Force of the European Society of Cardiology and Other Societies on Cardiovascular Disease Prevention in Clinical Practice (constituted by representatives of 10 societies and by invited experts)Developed with the special contribution of the European Association for Cardiovascular Prevention \& Rehabilitation (EACPR). Eur Heart J, 2016. 37(29): p. $2315-2381$.

[6]. Yang, C.W., et al., Subclinical Atherosclerosis Markers of Carotid Intima-Media Thickness, Carotid Plaques, Carotid Stenosis, and Mortality in Community-Dwelling Adults.Int J Environ Res Public Health, 2020. 17(13).

[7]. Zhao, Q., et al., LDL-C:HDL-C ratio and common carotid plaque in Xinjiang Uygur obese adults: a cross-sectional study. BMJ Open, 2018. 8(10): p. e022757.

[8]. Guo, X., et al., An update on overweight and obesity in rural Northeast China: from lifestyle risk factors to cardiometabolic comorbidities. BMC Public Health, 2014. 14: p. 1046.

[9]. Skinner, A.C., et al., Cardiometabolic Risks and Severity of Obesity in Children and Young Adults. N Engl J Med, 2015. 373(14): p. 1307-17.

[10]. Cai, L., et al., Prevalence, awareness, treatment, and control of dyslipidemia among adults in Beijing, China. J Atheroscler Thromb, 2012. 19(2): p. 159-68.

[11]. Valensi, P., et al., Atherogenic dyslipidemia and risk of silent coronary artery disease in asymptomatic patients with type 2 diabetes: a cross-sectional study. Cardiovasc Diabetol, 2016. 15(1): p. 104.

[12]. Packard, C.J., et al., Plasma lipoproteins and apolipoproteins as predictors of cardiovascular risk and treatment benefit in the PROspective Study of Pravastatin in the Elderly at Risk (PROSPER). Circulation, 2005. 112(20): p. 3058-65.

[13]. Endo, A., et al., Contributors to newly developed coronary artery disease in patients with a previous history of percutaneous coronary intervention beyond the early phase of restenosis. Intern Med, 2014. 53(8): p. 819-28.

[14]. Kunutsor, S.K., et al., Is High Serum LDL/HDL Cholesterol Ratio an Emerging Risk Factor for Sudden Cardiac Death? Findings from the KIHD Study. J Atheroscler Thromb, 2017. 24(6): p. 600-608.

[15]. Matsumoto, I., et al., Impact of low-density lipoprotein cholesterol/high-density lipoprotein cholesterol ratio on long-term outcome in patients undergoing percutaneous coronary intervention. Circ J, 2011. 75(4): p. 905-10.

[16]. Zhong, Z., et al., Assessment of the LDL-C/HDL-C ratio as a predictor of one year clinical outcomes in patients with acute coronary syndromes after percutaneous coronary intervention and drug-eluting stent implantation. Lipids Health Dis, 2019. 18(1): p. 40. 
[17]. Yu, Y., et al., A U-shaped association between the LDL-cholesterol to HDL-cholesterol ratio and all-cause mortality in elderly hypertensive patients: a prospective cohort study. Lipids Health Dis, 2020. 19(1): p. 238.

[18]. Liu, L., et al., Association of LDL-C/HDL-C Ratio With Stroke Outcomes Within 1 Year After Onset: A Hospital-Based Follow-Up Study. Front Neurol, 2020. 11: p. 408.

[19]. You, S., et al., LDL-C/HDL-C ratio and risk of all-cause mortality in patients with intracerebral hemorrhage. Neurol Res, 2016. 38(10): p. 903-8.

[20]. Shinkawa, Y., et al., Mathematical modeling for the prediction of cerebral white matter lesions based on clinical examination data. PloS one, 2019. 14(4): p. e0215142-e0215142.

[21]. Zhang, H., et al., Associations between Carotid Artery Plaque Score, Carotid Hemodynamics and Coronary Heart Disease. Int J Environ Res Public Health, 2015. 12(11): p. 14275-84.

[22]. Piepoli, M.F., et al., 2016 European Guidelines on cardiovascular disease prevention in clinical practice: The Sixth Joint Task Force of the European Society of Cardiology and Other Societies on Cardiovascular Disease Prevention in Clinical Practice (constituted by representatives of 10 societies and by invited experts)Developed with the special contribution of the European Association for Cardiovascular Prevention \& Rehabilitation (EACPR). Eur Heart J, 2016. 37(29): p. $2315-2381$.

[23]. Bhanpuri, N.H., et al., Cardiovascular disease risk factor responses to a type 2 diabetes care model including nutritional ketosis induced by sustained carbohydrate restriction at 1 year: an open label, non-randomized, controlled study. Cardiovasc Diabetol, 2018. 17(1): p. 56.

[24]. Valensi, P., et al., Atherogenic dyslipidemia and risk of silent coronary artery disease in asymptomatic patients with type 2 diabetes: a cross-sectional study. Cardiovasc Diabetol, 2016. 15(1): p. 104.

[25]. Lou, Y., et al., LDL-cholesterol to HDL-cholesterol ratio discordance with lipid parameters and carotid intima-media thickness: a cohort study in China. Lipids Health Dis, 2020. 19(1): p. 141.

[26]. Qin, P., et al., Low-Density Lipoprotein Cholesterol/High-Density Lipoprotein Cholesterol Ratio and Carotid Intima-Media Thickness: A Cohort Study in China. Lipids, 2021. 56(1): p. 59-68.

[27]. Enomoto, M., et al., LDL-C/HDL-C Ratio Predicts Carotid Intima-Media Thickness Progression Better Than HDL-C or LDLC Alone. J Lipids, 2011. 2011: p. 549137.

[28]. Varma, V., et al., Serum Total Sialic Acid and Highly Sensitive C-reactive Protein: Prognostic Markers for the Diabetic Nephropathy. J Lab Physicians, 2016. 8(1): p. 25-9.

[29]. Zhong, Z., et al., Assessment of the LDL-C/HDL-C ratio as a predictor of one year clinical outcomes in patients with acute coronary syndromes after percutaneous coronary intervention and drug-eluting stent implantation. Lipids Health Dis, 2019. 18(1): p. 40.

[30]. Umeda, T., et al., Low-Density Lipoprotein Cholesterol Goal Attainment Rates by Initial Statin Monotherapy Among Patients With Dyslipidemia and High Cardiovascular Risk in Japan- A Retrospective Database Analysis. Circ J, 2018. 82(6): p. 1605-1613.

[31]. Manninen, V., et al., Joint effects of serum triglyceride and LDL cholesterol and HDL cholesterol concentrations on coronary heart disease risk in the Helsinki Heart Study. Implications for treatment. Circulation, 1992. 85(1): p. 37-45.

[32]. Katakami, N., et al., Usefulness of lipoprotein ratios in assessing carotid atherosclerosis in Japanese type 2 diabetic patients. Atherosclerosis, 2011. 214(2): p. 442-7. 
[33]. Wu, T.W., et al., Associations of Cardiovascular Risk Factors with Carotid Intima-Media Thickness in Middle-Age Adults and Elders. J Atheroscler Thromb, 2017. 24(7): p. 677-686.

[34]. Nimkuntod, P. and P. Tongdee, Plasma Low-Density Lipoprotein Cholesterol/High-Density Lipoprotein Cholesterol Concentration Ratio and Early Marker of Carotid Artery Atherosclerosis. J Med Assoc Thai, 2015. 98 Suppl 4: p. S58-63.

[35]. Qin, P., et al., Low-Density Lipoprotein Cholesterol/High-Density Lipoprotein Cholesterol Ratio and Carotid Intima-Media Thickness: A Cohort Study in China. Lipids, 2021. 56(1): p. 59-68.

\section{Figures}

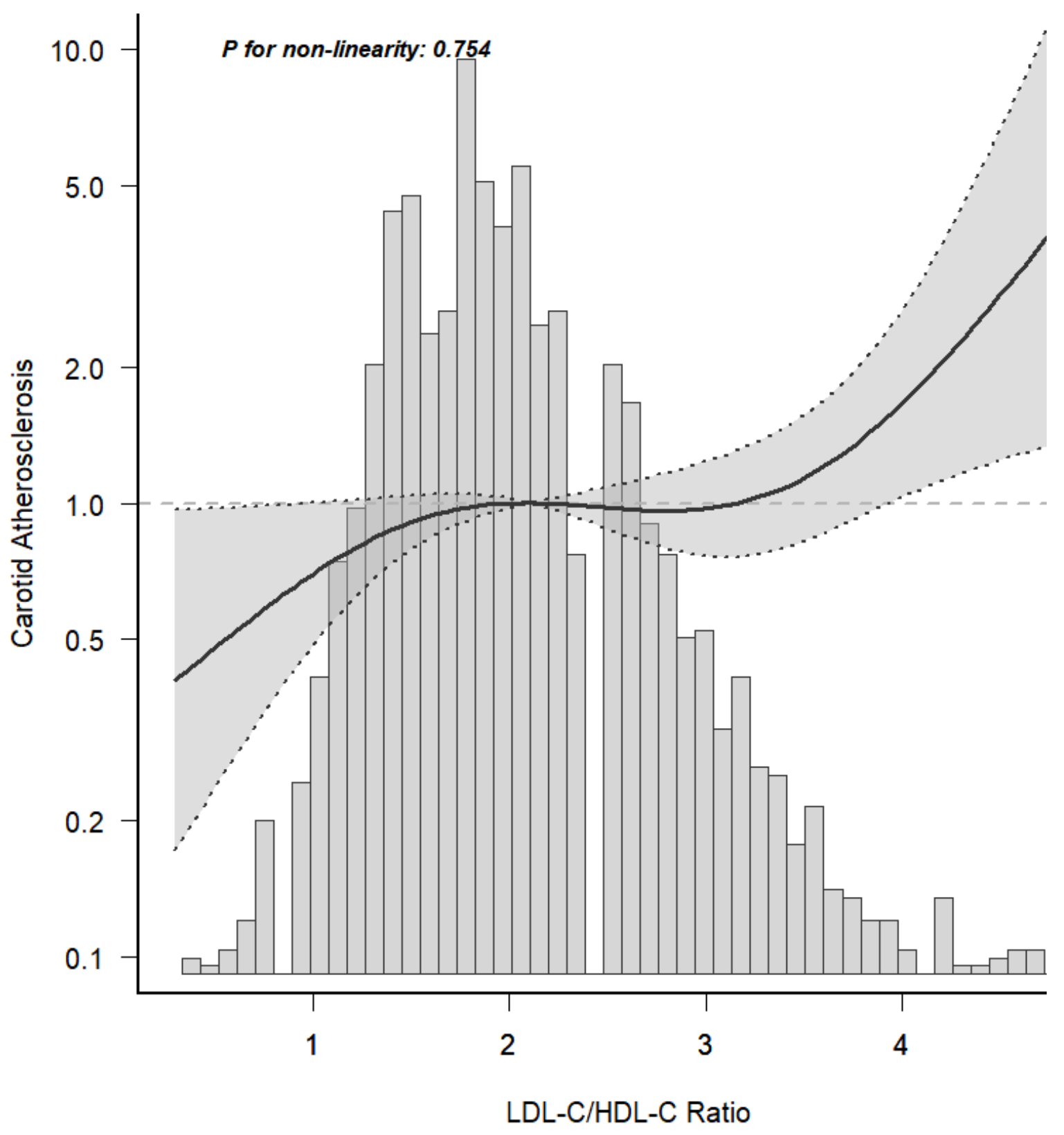

Figure 1

LDL-C/HDL-C ratio level 


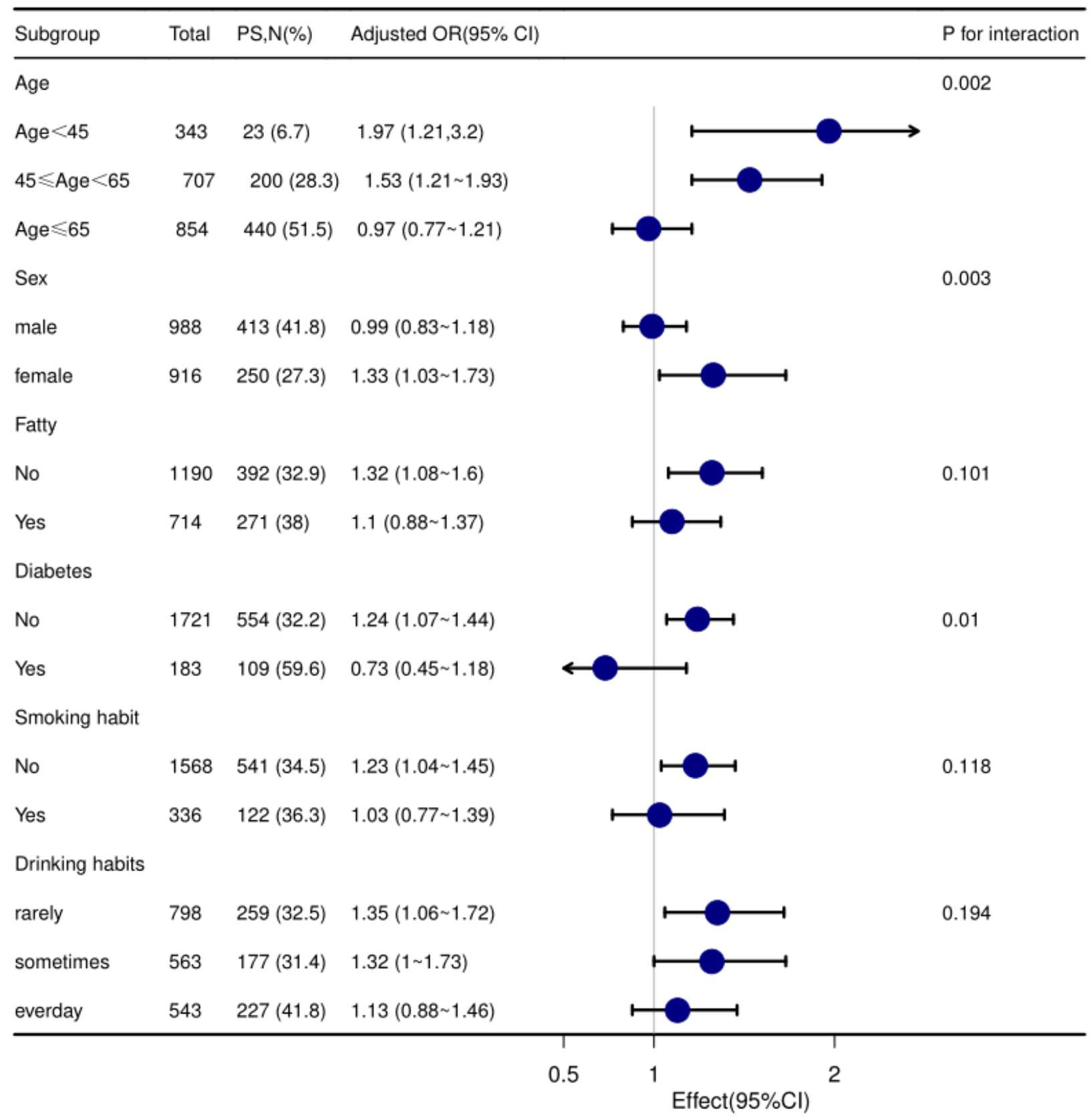

\section{Figure 2}

Association between baseline LDL-C/HDL-C ratio and the risk of Carotid Atherosclerosis in the subgroup analyses 